\title{
Virtual Reality in Orthopaedics
}

\section{Is It a Reality?}

\author{
Jay D. Mabrey MD, Karl D. Reinig PhD, \\ W. Dilworth Cannon MD
}

Published online: 18 June 2010

(C) The Association of Bone and Joint Surgeons( 2010 in-depth review of a VR simulator for knee arthroscopy is also presented.

Where are we now? The number of papers specific to orthopaedics and VR is limited. VR is used effectively in other specialties, especially general surgery. VR simulators are readily available for shoulder and knee arthroscopy but not as well incorporated into training curricula.

Where do we need to go? VR technology is available today for training programs. Integration of VR into the orthopaedic curriculum will save time in the OR, reduce operative errors, and improve the resident's overall educational experience. The public will expect their surgeons to train on these simulators.

How do we get there? Orthopaedic training programs should take advantage of the commercially available VR simulators for orthopaedic procedures and incorporate them into their training curricula. This effort could be led by the American Academy of Orthopaedic Surgeons (AAOS) and the American Board of Orthopaedic Surgery (ABOS), two of the primary sponsors of a major study in the effectiveness of VR simulators for knee arthroscopy.

\section{Introduction}

On January 15, 2009, Captain Chesley Sullenberger III landed an Airbus 320 with two failed engines in the middle of the Hudson River in New York City with no loss of life [6]. As is true for all major airline pilots, he had previously rehearsed this scenario several times in a high fidelity flight simulator. Ironically, when it was time to return to the skies, Capt. Sullenberger was required to perform three takeoffs and landings in the same simulator, one of those simulating an engine failure [16]. While there are no such requirements for surgeons who spend time away from the 
operating room, several general surgery programs have been using virtual reality (VR) simulators for laparoscopic training for several years.

Repetition and rehearsal are also important to orthopaedic surgeons. Several studies report steep learning curves associated with many newly introduced orthopaedic procedures. One study examining the learning curve associated with endoscopic anterior scoliosis surgery in 100 consecutive patients demonstrated experience resulted in "statistically significant improvements" in operative time, X-ray irradiation time, and blood loss [10]. Another study documented substantial improvement in pedicle screw fixation after the first 80 screws inserted by a spinal surgery fellow [12]. Rong et al. [21] noted surgeons learning to perform microendoscopic discectomy (MED) became proficient at the procedure after 10 to 20 cases.

Several factors are driving the push toward VR simulators in orthopaedics. Surgery is an apprenticeship technique that requires additional time for training in the operating room. In one study, orthopaedic residents took over 40 minutes longer to repair an anterior cruciate ligament tear than did the faculty surgeon [8]. Training residents is an expensive endeavor for the supporting hospital with some estimates reaching $\$ 48,000$ per resident per year in operating room time alone [5]. Recently, Britain's Chief Medical Officer, Sir Liam Donaldson, was quoted as saying "If I had to have my gall bladder out I would rather it was done by someone who had already done 100 in a simulator," and then cited flight simulator training as one of the major contributors to Capt. Sullenberger's safe landing on the Hudson [18].

We asked (1) how VR has worked for other surgical specialties; (2) what VR solutions are available for orthopaedics; and (3) should VR simulation become part of the orthopaedic curriculum?

\section{Materials and Methods}

We conducted an informal review of the Pub Med database from the National Library of Medicine beginning with relevant search terms and then refining the search to cull out specific topics. The literature review began with the search terms "virtual reality" AND "surgery" yielding 1025 citations spanning 1992 to 2009. This subset, VR+Surgery, was then searched using "orthopaedic" OR "orthopedic" OR "fracture" OR "spine" OR "hip" OR "knee" OR "shoulder", yielding 232 articles from 1994 to 2009. Each title and abstract was then examined as to whether the article was appropriate for orthopaedics, giving a subset of 48 articles from 1995 to 2009.

The Task Force on Virtual Reality was established in 1996 as a collaboration between the American Academy of
Orthopaedic Surgeons, the Arthroscopy Association of North America, the American Board of Orthopaedic Surgeons, and Touch of Life Technologies [11] to explore the feasibility of developing a VR surgical simulator for arthroscopy of the knee. One of the authors (JDM) chaired that task force until this year when its mission was completed; the second author (KDR) was a member of that task force and one of the developers of the hardware and software for the simulator; and the third author (WDC) served on the task force as well as leading the content development group (CDG) for the educational and evaluation arm of the project. The CDG was initially comprised of five orthopaedic surgeons recognized as experts in arthroscopy of the knee.

The Knee Arthroscopy Surgical Trainer (KAST) developed by the American Academy of Orthopaedic Surgeons' Task Force on Virtual Reality is a system designed to provide an intuitive and autonomous experience for the orthopaedic resident. Given our involvement with that task force, we are familiar with the details of the development the KAST. KAST consists of a hardware component supported by proprietary software and a didactic component delivered on one of two monitors positioned in front of the trainee (Fig. 1). The core of the hardware component is a pair of high-fidelity haptic, or force feedback, devices that recreate the feel of the arthroscope and the probe within the virtual knee. The workspace can be raised or lowered electronically to accommodate a wide range of statures, sitting or standing.

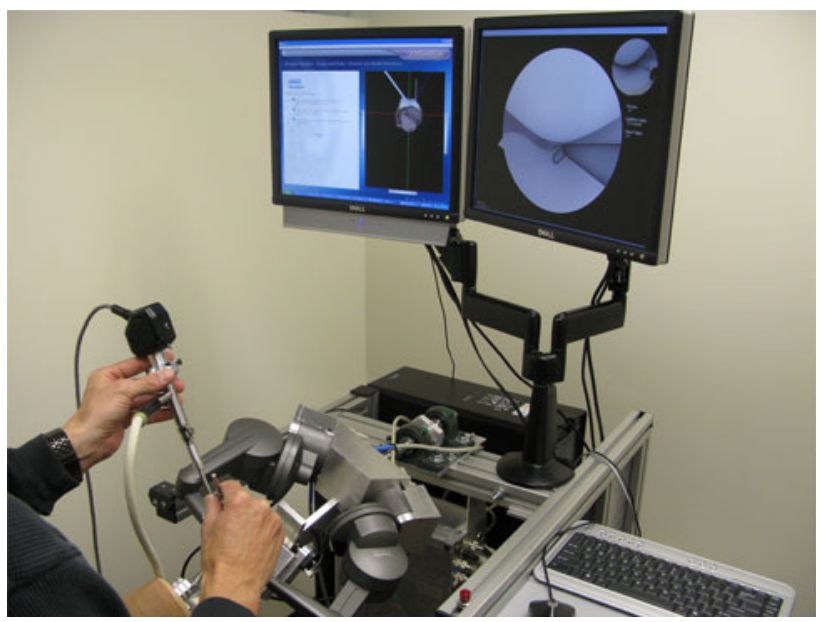

Fig. 1 The Knee Arthroscopy Surgical Trainer (KAST). The "Mentor" screen is on the trainee's left and the "camera view" is on the right. The trainee is holding the arthroscope and camera in his left hand and a simple probe in the right. Each is attached to a haptic, or force feedback, device that recreates the touch and feel of the instruments interacting with the structures of the knee. Both haptic devices are mounted on a universal joint attached to a surrogate leg that allows the trainee to flex and extend the virtual knee as well as apply varus and valgus forces. 
To complete the feeling of performing a real arthroscopy, the trainee must manipulate a surrogate leg via flexion and extension of the knee and the application of varus and valgus forces. The simulator switches seamlessly between a right and a left knee, forcing the trainee to be ambidextrous with respect to the camera and probe. The images appearing on the monitor screen to the trainee's right are generated by a proprietary program that recreates threedimensional models of the structures within the knee based upon data from the Visible Human Project [1] or similar but higher-resolution cryosectioned data. These structures interact with the virtual probe and arthroscope, just as the cartilage and synovial lining of a real knee would interact. Techniques developed by Touch of Life Technologies alter the morphology, posture, and pathologies displayed from the data, giving a limitless supply of virtual patients.

The didactic component of KAST, known as the "Mentor," is displayed to the trainee on the left monitor. The Mentor was developed in close consultation with the CDG led by one of the authors (WDC). After logging on to KAST, the Mentor directs the trainee through a series of brief lessons demonstrating how to run the simulator. The Mentor program utilizes Hypertext Markup Language (HTML) to display text, audio, images, and video. In addition, the Mentor displays Interactive Anatomic Animations (IAA) utilizing texture mapped polygonal models to render complex anatomic scenes that may be altered by the trainee's actions. After that, the trainee is instructed on how to perform a complete diagnostic sweep of the knee with the arthroscope only, followed by a sweep with a probe in the opposite hand. The Mentor continuously records all activity generated by the trainee and then provides sophisticated feedback concerning the trainee's progress.

Throughout the session, trainees must achieve proficiency while following the instructions from the Mentor program (Fig. 2). The Mentor requires the trainee to score $100 \%$ on each step before proceeding to the next task. When they have successfully completed all required tasks, they do the entire procedure on their own and then one last time with the entire procedure timed. There is a "God'seye view" of the outside of the knee that shows where the arthroscope and the probe are with respect to the knee anatomy to assist the trainee with triangulation (Fig. 2). This view is not available once the trainee advances to the timed tasks. KAST will continue to evolve through software updates to include meniscal resection and ACL repair. KAST has been designed so that the same hardware can be used for simulation of shoulder arthroscopy.

We are currently involved a study asking whether postgraduate year three (PGY-3) residents trained on the simulator will complete a diagnostic arthroscopy on an actual patient in less time with less damage to the patient's tissue compared with control residents who learned arthroscopy through the residency's established training program [7]. As part of the evaluation process, each resident will complete a live surgical case which will be recorded on digital versatile disc (DVD) and the CDG, now expanded to ten arthroscopy experts, will grade the performance of the experimental and control residents in a blinded fashion. The CDT will employ an elaborate
Fig. 2 The "Mentor" screen for KAST displays a set of instructions or tasks for the trainee on the left side and a "God's-eye" view on the right to help orient the trainee during the initial phases of instruction. Later, during timed tasks, the "God's-eye" view is not available.

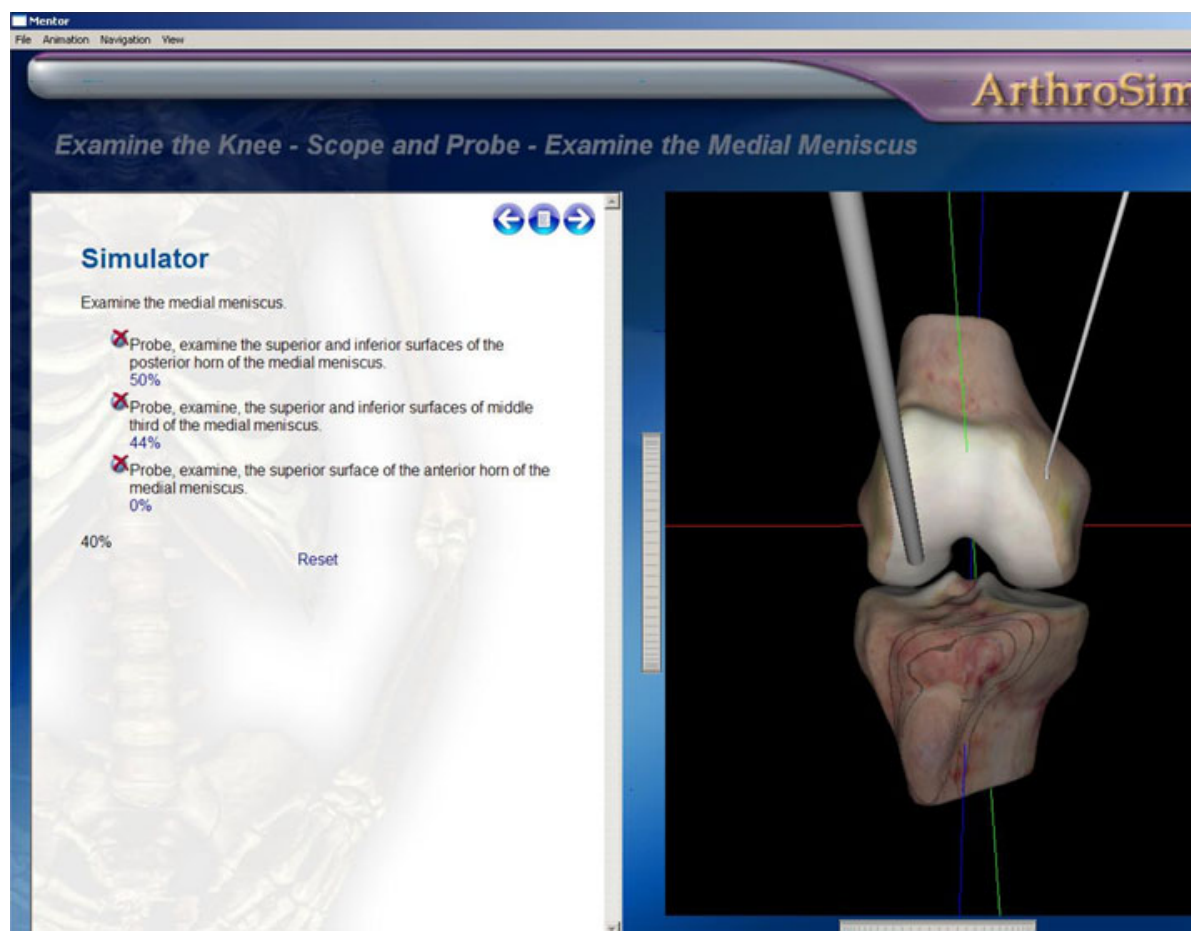


checklist of 51 points with a 4 point Likert scale for each scored item. Each item is also rated in importance to other steps and tasks during the diagnostic sweep of the knee. The aim is to have an interrater reliability such that $r$ equals at least 0.6. The grading process will not occur until after all experimental sites have completed the study. The CDT has scheduled practice grading sessions for its members in the near future.

Three test sites have completed the experimental protocol: The University of California at San Francisco (UCSF), Northwestern University in Chicago, and The Hospital for Joint Disease in New York City. The latest test site is the Harvard orthopaedic program at Mass General in Boston. Next in line are the University of Minnesota and the University of Iowa, thus completing a total of eight institutions by the spring of 2011 .

\section{Results}

Of the subset of 48 relevant orthopaedic articles from 1995 to 2009 found in the informal literature review, only 23 articles dealt with specific simulators, with the rest being more general reviews of this topic. Of these 23 articles, only 16 dealt with specific simulators with the rest covering principles of VR training as it related to orthopaedics. These broke down into nine papers about knee arthroscopy simulators (1995-2006), four involving shoulder simulators (19992008), and three with fractures (2007-2008.) For comparison, there were 246 citations for laparoscopic virtual reality simulation out of the original 1025 citations (1992-2009).

In one of the first prospective, randomized, blinded studies of the use of VR surgical simulation to train skills and reduce error risk in the operating room, Seymour et al. [22], demonstrated the use of VR surgical simulation substantially improved OR performance. They assigned 16 general surgery residents to one of two groups: VR-trained or non-VR-trained. The VR-trained group worked on the simulators until they had achieved "expert criterion levels established by experienced laparoscopists" [22]. The nonVR-trained group served as the control. An experienced laparoscopic surgeon then assisted them in performing a cholecystectomy. Two more experienced laparoscopists blinded to each subject's training reviewed videotapes of each surgery. As one would expect, there were no differences in baseline skills of the two groups. However, the VR-trained residents performed their dissections substantially faster while the non-VR-trained residents were more likely to fail to progress in the surgery. They were also much more likely to injure the gallbladder or burn nontarget tissue [22].

One of the major factors responsible for the success of VR laparoscopic simulators is what Gallagher et al. [9], refer to as "attentional capacity threshold" or ACT. ACT is a result of humans being able to comprehend and assess only a finite amount of information at one time, with any additional information being ignored once that limit has been reached. The master surgeon has a surplus of attentional capacity because he/she uses less of their attentional resources in monitoring the position of their hands, the condition of the patient, and making operative judgments. The novice surgeon has no such surplus because his/her surgical skills are not as automatic as those of the master surgeon. Pretraining novices on a VR simulator provides an attentional capacity buffer at the time of surgery because pretrained novices are not spending as much time monitoring their performance as untrained novices [9].

Laparoscopic VR simulators have been available for some time now and have been incorporated into the training programs of most general surgery residencies, thus there are several randomized clinical trials documenting their effectiveness. Gurusamy et al. [14] reviewed 23 randomized control trials of VR laparoscopic simulators that included 612 participants. They reported that VR laparoscopic training decreased the time for task completion and increased overall accuracy in comparison with control subjects who had not undergone VR training.

Pedowitz, Esch, and Snyder [20] evaluated a VR shoulder arthroscopy simulator (Mentice Corp, Gothenberg, Sweden) using a standardized skills assessment algorithm looking for differences among three groups: medical students, orthopaedic residents, and orthopaedic faculty at a shoulder arthroscopy course. They found much greater consistency in results from the experienced surgeons compared to the students and residents.

Srivastava et al. [23] later studied the construct validity of the same shoulder arthroscopy simulator by demonstrating its ability to differentiate among novice, intermediate, and expert shoulder arthroscopists. Novices had no experience, intermediates had assisted or performed up to 50 shoulder arthroscopies within the last five years, and experts had assisted or performed more than 50 shoulder arthroscopies. The experts consistently required less time to manipulate the arthroscopic hook but only in the third trial did this difference achieve significance [23]. The experts also performed better on scope manipulation than the other groups, yet there was no difference in the three groups' ability to identify anatomic structures. The authors hypothesized that some of the anatomy was not represented realistically enough.

While the previous studies confirmed that VR simulators could distinguish novice from expert in certain tasks, it was only a single time point evaluation. Gomoll et al. [11] took the next step and studied 10 orthopaedic residents as novices, and then three years later after they had completed three additional rotations in sports medicine and 
participated in an average of 60 shoulder arthroscopies. In this study, the participants were required to locate and probe a simulated target within the shoulder joint and were graded on time to completion, number of collisions, average probe velocity, and distance the probe tip traveled. The residents improved in all four parameters [11].

\section{Discussion}

The incorporation of virtual reality technology into the curricula of any one orthopaedic training program depends upon the vision of individual program directors. It is clear, however, that VR technology, when applied to the education of residents in general surgery programs, does have a positive impact on their training [2, 3, 13, 17, 24, 25].

Reports of virtual reality simulation of orthopaedic procedures are far less common than those for laparoscopic procedures. Heng et al. [15] described a VR based training system for knee arthroscopy based upon the Chinese Visible Human data set. It provided haptic feedback to the user through a Phantom device similar to that used in the KAST. McCarthy et al. [19] developed a cost-effective VR simulator, the Sheffield Knee Arthroscopy Training System (SKATS), using passive feedback from physical bones. They reported that it could differentiate between expert and novice surgeons [19]. Bliss et al. [4] tested a group of ten psychology graduate students on the Procedicus Virtual Arthroscopy knee trainer (Mentice Corp.) and reported that they made steady improvements in task times with each session.

Widespread implementation of surgical simulators faces significant challenges. First, the current crop of simulators, while quite advanced, can only address a limited range of surgical skills and sites. The next generation of simulators will employ a "universal platform" that enables the user to switch from arthroscopy of the knee to the shoulder or the hip. Additional task simulations such as anterior cruciate ligament reconstruction will take more time to implement. Second, cost remains a barrier to adoption only as long as orthopaedic departments isolate their teaching functions from the business of running a hospital operating room. The evolving health care climate will almost certainly require educators and administrators to cooperate to make the best use of their resources. The experience with laparoscopic simulators confirms that hospitals that support the use of simulators will recoup that investment by reducing time in the operating room. Third, just as laparoscopic simulators took some time to be validated, orthopaedic simulators will reach that point as well. KAST is in the middle of an extensive validation study spearheaded by one of the authors (WDC) with results expected to be published within the next year. Another author (JDM) is working with the Department of Surgery at his institution to incorporate knee simulators into a planned expansion of their surgical skills laboratory. Locating the simulators in a supervised environment will also address the problem of maintaining these rather delicate devices at the hands of overly enthusiastic orthopaedic residents.

Where are we now? As Cannon et al. [7], have noted, the Orthopaedic Learning Center of the American Academy of Orthopaedic Surgeons (AAOS) in Rosemont, IL, offers state of the art arthroscopic training, but this training depends upon cadaveric specimens that vary considerably in terms of anatomy and internal pathology. Unfortunately, very few programs have formal arthroscopic training on cadavers or models as part of their curricula, leaving most orthopaedic trainees to acquire their arthroscopic skills by apprenticeship learning [7].

Where do we need to go? What is missing from the equation is a nationwide standard that all orthopaedic training programs can agree upon. KAST has been supported by the American Academy of Orthopaedic Surgeons (AAOS), the Arthroscopy Association of North America (AANA), and the American Board of Orthopaedic Surgery (ABOS) since its inception over ten years ago. As the cost of hardware has fallen over the years, the price of one KAST unit has dipped below \$100,000 USD, making it affordable for many training programs. The system is already up and running at several training programs including Duke University Medical Center, The University of Michigan, The Pan Am Clinic in Winnipeg, Brooke Army Medical Center in San Antonio, Banner Health in Phoenix, and The University of Colorado at Denver.

Where do we need to go? It is reasonable to expect that once KAST has been validated as an effective training tool hospitals and training programs will incorporate it into their curricula as one of the standards with which to improve quality of patient care. As the AAOS and ABOS were involved with KAST's inception, they should insist upon KAST as one of the training standards for orthopaedic residents. The successful experience of general surgery programs with a variety of VR simulators also suggests that programs should consider the use of the Mentice shoulder and knee simulators as well. Once incorporated into the basic training curriculum, the next logical step to using these and newly developed simulators is to evaluate orthopaedic surgeons for board certification.

\section{References}

1. Ackerman MJ. The Visible Human Project: a resource for anatomical visualization. Stud Health Technol Inform. 1998;52 Pt 2:1030-1032.

2. Aggarwal R, Ward J, Balasundaram I, Sains P, Athanasiou T, Darzi A. Proving the effectiveness of virtual reality simulation 
for training in laparoscopic surgery. Ann Surg. 2007;246:771779 .

3. Ahlberg G, Enochsson L, Gallagher AG, Hedman L, Hogman C, McClusky DA, III, Ramel S, Smith CD, Arvidsson D. Proficiency-based virtual reality training significantly reduces the error rate for residents during their first 10 laparoscopic cholecystectomies. Am J Surg. 2007;193:797-804.

4. Bliss JP, Hanner-Bailey HS, Scerbo MW. Determining the efficacy of an immersive trainer for arthroscopy skills. Stud Health Technol Inform. 2005;111:54-56.

5. Bridges M, Diamond DL. The financial impact of teaching surgical residents in the operating room. Am J Surg. 1999;177:28-32.

6. Burke K, Donohue P, Siemaszko C. US Airways airplane crashes in Hudson River - Hero pilot Chesley Sullenberger III saves all aboard. New York Daily News. January 16, 2009. http://www.ny dailynews.com/news/2009/01/15/2009-01-15_us_airways_airplane_ crashes_in_hudson_ri.html?page $=1$

7. Cannon WD, Eckhoff DG, Garrett WE, Jr., Hunter RE, Sweeney HJ. Report of a group developing a virtual reality simulator for arthroscopic surgery of the knee joint. Clin Orthop Relat Res. 2006;442:21-29.

8. Farnworth LR, Lemay DE, Wooldridge T, Mabrey JD, Blaschak MJ, DeCoster TA, Wascher DC, Schenck RC, Jr. A comparison of operative times in arthroscopic ACL reconstruction between orthopaedic faculty and residents: the financial impact of orthopaedic surgical training in the operating room. Iowa Orthop J. 2001;21:31-35.

9. Gallagher AG, Ritter EM, Champion H, Higgins G, Fried MP, Moses G, Smith CD, Satava RM. Virtual reality simulation for the operating room: proficiency-based training as a paradigm shift in surgical skills training. Ann Surg. 2005;241:364-372.

10. Gatehouse SC, Izatt MT, Adam CJ, Harvey JR, Labrom RD, Askin GN. Perioperative aspects of endoscopic anterior scoliosis surgery: the learning curve for a consecutive series of 100 patients. J Spinal Disord Tech. 2007;20:317-323.

11. Gomoll AH, O’Toole RV, Czarnecki J, Warner JJ. Surgical experience correlates with performance on a virtual reality simulator for shoulder arthroscopy. Am J Sports Med. 2007;35:883-888.

12. Gonzalvo A, Fitt G, Liew S, de la Harpe D, Turner P, Ton L, Rogers MA, Wilde PH. The learning curve of pedicle screw placement: how many screws are enough? Spine (Phila Pa 1976). 2009;34:E761-765.

13. Grantcharov TP, Bardram L, Funch-Jensen P, Rosenberg J. Learning curves and impact of previous operative experience on performance on a virtual reality simulator to test laparoscopic surgical skills. Am J Surg. 2003;185:146-149.
14. Gurusamy K, Aggarwal R, Palanivelu L, Davidson BR. Systematic review of randomized controlled trials on the effectiveness of virtual reality training for laparoscopic surgery. $\mathrm{Br} \mathrm{J}$ Surg. 2008;95:1088-1097.

15. Heng PA, Cheng CY, Wong TT, Wu W, Xu Y, Xie Y, Chui YP, Chan KM, Leung KS. Virtual reality techniques. Application to anatomic visualization and orthopaedics training. Clin Orthop Relat Res. 2006;442:5-12.

16. Klein M. NICE, SULLY - NOW TRY IT ALL AGAIN. The New York Post. March 1, 2009. http://www.nypost.com/p/news/ regional/item_AlI97WsXnKUJuzxQvF4xGJ;jsessionid=5E3F206 1AEAEA7DFFA8BEC1043EFDF44

17. Larsen CR, Soerensen JL, Grantcharov TP, Dalsgaard T, Schouenborg L, Ottosen C, Schroeder TV, Ottesen BS. Effect of virtual reality training on laparoscopic surgery: randomised controlled trial. BMJ. 2009;338:b1802.

18. Laurance J. Surgeons should train on simulators, like pilots. The Independent. March 16, 2009. http://www.independent.co.uk/lifestyle/health-and-families/health-news/surgeons-should-train-onsimulators-like-pilots-1645737.html

19. McCarthy AD, Moody L, Waterworth AR, Bickerstaff DR. Passive haptics in a knee arthroscopy simulator: is it valid for core skills training? Clin Orthop Relat Res. 2006;442:13-20.

20. Pedowitz RA, Esch J, Snyder S. Evaluation of a virtual reality simulator for arthroscopy skills development. Arthroscopy. 2002;18:E29.

21. Rong LM, Xie PG, Shi DH, Dong JW, Liu B, Feng F, Cai DZ. Spinal surgeons' learning curve for lumbar microendoscopic discectomy: a prospective study of our first 50 and latest 10 cases. Chin Med J (Engl). 2008;121:2148-2151.

22. Seymour NE, Gallagher AG, Roman SA, O'Brien MK, Bansal VK, Andersen DK, Satava RM. Virtual reality training improves operating room performance: results of a randomized, double-blinded study. Ann Surg. 2002;236:458-463; discussion 463-454.

23. Srivastava S, Youngblood PL, Rawn C, Hariri S, Heinrichs WL, Ladd AL. Initial evaluation of a shoulder arthroscopy simulator: establishing construct validity. J Shoulder Elbow Surg. 2004;13: 196-205.

24. Stefanidis D, Korndorffer JR, Jr., Sierra R, Touchard C, Dunne JB, Scott DJ. Skill retention following proficiency-based laparoscopic simulator training. Surgery. 2005;138:165-170.

25. Verdaasdonk EG, Dankelman J, Lange JF, Stassen LP. Transfer validity of laparoscopic knot-tying training on a VR simulator to a realistic environment: a randomized controlled trial. Surg Endosc. 2008;22:1636-1642. 